\title{
Hydration Simulations of a Carbon Nanotube, Immersed in Water, according to the 3-Attractor Water Model
}

\author{
Yuri Bushuev ${ }^{1, *}$, Svetlana Davletbaeva ${ }^{2}$ and Francis F. Muguet ${ }^{3, *}$ \\ ${ }^{1}$ Ivanovo State University of Chemistry and Technology, pr. F. Engel'sa, 7, Ivanovo, Russia, 153460 \\ ${ }^{2}$ Institute of Chemistry of Solutions RAS, Akademicheskaya, 1, Ivanovo, Russia, 153045 \\ ${ }^{3}$ E.N.S.T.A., 32 Boulevard Victor 75739 Paris Cedex France \\ *Authors to whom correspondence should be addressed. E-mail: bushuev@isuct.ru, muguet@ensta.fr
}

Received: 15 December 2003 / Accepted: 7 April 2005 / Published: 8 April 2005

\begin{abstract}
MC simulations of a set of zigzag $((9,0)-(14,0))$ and armchair $((6,6)-(10,10))$ carbon nanotubes immersed in water have been carried out in an NpT-ensemble (512 water molecules, $\mathrm{p}=1$ bar, $\mathrm{T}=298 \mathrm{~K}$ ). Intermolecular interactions were described by BMW potential according to which, besides the well-known linear water dimer bifurcated and inverted water dimers are metastable. In all cases, it was found that there are large periodic fluctuations of water occupancy inside the nanotubes. Decrease in the size of the nanotube diameter leads to a significant destruction of the H-bond network, and to a bifucarted dimer population increase. Inverted dimer concentration relationship with the nanotube diameter is more complicated. Population maximum for inverted dimers occurs for diameters of 10$11 \AA$. Water features different intermolecular structures not only inside carbon nanotubes but also in the outer first hydration shells. The amount of bifurcated and inverted dimers is significantly more important in the first hydration shell than in bulk water.
\end{abstract}

Keywords: water, water structure, carbon nanotube, hydration, H-bonds, Monte-Carlo simulation, intermolecular interactions, water potential. 


\section{Introduction}

A single-walled carbon nanotube $(\mathrm{CN})$ is a nanoscale tube formed by a single cylindrical layer of carbon atoms. Nanotubes feature diameters of a few nanometers and lengths up to 100 micrometers. They form extremely thin wires. The intermolecular structure of nanotubes can be formed by wrapping around a single atomic layer from graphite sheets, along a certain direction, and this direction determines the diameter and chirality of the nanotubes.

Experimental and theoretical studies have determined that those nanometer-sized CNs possess novel electronic characteristics, which can be either metallic or semi-conducting, depending on their radius or chiralities. Mechanical deformations or chemical doping can induce strong changes in conductance. Such changes can be easily detected by electron current signals, and these properties are making CNs very attractive components of extremely small sensor devices which are sensitive to their chemical and mechanical environments.

Indian researchers have recently positioned fixed CNs within a liquid flow and in this way generated voltage [1]. The authors are proposing that this technology could provide a way to manufacture fluid-flow sensors as well as voltage sources for nanoscale medical research and applications. The voltage which the nanotubes generate, is relatively high, even at low flow speeds of $1 \mu \mathrm{m} / \mathrm{s}$. It is quite interesting to notice that a polar liquid such as water provides much higher voltages than non-polar liquids. The voltage created can be used both as a current source and as a control mechanism.

In yet another study [2], it has been shown that small proteins can be entrapped into the inner channel of opened nanotubes by simple adsorption. Carbon nanotubes may play a dual role, both as immobilization matrices and as mediators, allowing for the development of a third generation of biosensor systems, with good overall analytical characteristics.

Studies $[3,4]$ have been devoted to the investigation of $\mathrm{CN}$ properties related to their application as chemical sensors. Upon exposure to gaseous molecules such as $\mathrm{NO}_{2}$ or $\mathrm{NH}_{3}$, the electrical resistance of a semiconducting CNs found to dramatically increase or decrease [3].

In order to design sensors that may be selectively sensitive to specific physicochemical characteristics of a liquid, and with specific molecules in solution, it is crucial to understand the nature of interaction of a carbon nanotube with its surrounding solvent bath, and how properties are modified.

Water plays the most important role as a solvent. This liquid features rather unique physical, chemical, and thermodynamic properties. Explanation of the properties of liquid water is still a matter of intense research and many controversies. Many structural properties of liquid water at the mesoscopic level are still unknown.

According to molecular dynamic simulations studies of a $(6,6)$ carbon nanotube immersed in water [5], it has been concluded that hydrophobic channels may retain a significant water content, and that small variations of the nanotube water interactions may lead to large modifications in the water occupancy of the channel, while two-state transitions between empty and filled states are being observed. 
Many phenomenological theories have been presented about water structure; all are based more or less on the idea that the hydrogen bond is the only type of interaction between two water molecules. All these theories have not been very successful. Our current approach follows the 3 -attractors (3A) water model, proposed by Muguet [6-8]. According to the 3A model, one must consider also that the inverted water dimer (ID) and the bifurcated water dimer (BD) are local minima instead of saddle points when evaluating the isolated water dimer potential energy surface (PES), as well as the effective 2-body water intermolecular PES. To each PES well corresponds an attractor, that each water dimer trajectory may explore dynamically, jumping from one attractor to another one. It is not a 3-state static model. The 3A model PES comprises one global minimum and two local minima, corresponding to the following metastable geometries (see Fig. 1).
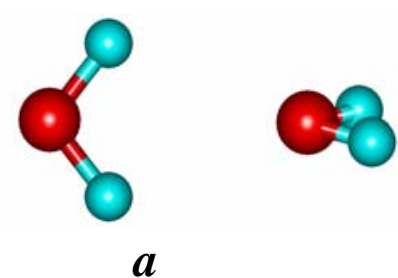

a

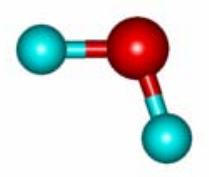

$\boldsymbol{b}$

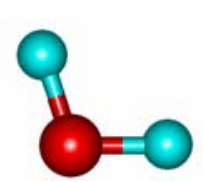

$b$
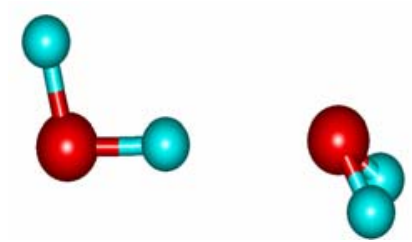

C

Figure 1. Linear $(a)$, inverted $(b)$, and bifurcated $(c)$ water dimer.

We recently proposed a new effective additive pair potential [8], called BMW (Bushuev-MuguetWater) built by modifying the well-known SPC/E potential [9] while adding Gaussian functions in order to describe the shape of the PES in regions corresponding to inverted and bifurcated dimer-type 2-body interactions. The results of simulations of water properties with the new BMW potential in a wide range of state parameters are indicating a surprisingly good agreement with calculated and experimental thermodynamical and structural properties of liquid water. In further studies, we are planning to improve and refine the BMW potential. We found that there is a significant population of inverted and bifurcated dimer-type interactions that are linking together water molecules in complex networks, that also include the more familiar linear H-bond type interaction. The population of inverted and bifurcated dimer essentially increases both with temperature and pressure.

The aims of the present investigation is to study the molecular mechanisms of CN hydration, and the changing character of structural properties of water in and outside the $\mathrm{CN}$.

\section{Potential and simulation details}

We investigated the hydration of sets of carbon nanotubes with different chiralities (Table 1) immersed in liquid water using Monte-Carlo computer simulation method.

There were fragments of nanotube and 512 water molecules in a cubic elementary cubic cell taken with periodic boundary conditions. The symmetry center of the $\mathrm{CN}$ was in the geometrical center of cell (Fig. 2). 
Table 1. Parameters of investigated CNs.

\begin{tabular}{|c|c|c|c|c|c|c|}
\hline $\begin{array}{l}\text { Zigzag tubes, } \\
\text { Length is } 9.3 \AA\end{array}$ & $(9,0)$ & $(10,0)$ & $(11,0)$ & $(12,0)$ & $(13,0)$ & $(14,0)$ \\
\hline Diameter, Å & 7.12 & 7.86 & 8.65 & 9.42 & 10.2 & 11.0 \\
\hline $\begin{array}{l}\text { Armchair tubes, } \\
\text { Length is } 11.2 \AA\end{array}$ & $(6,6)$ & $(7,7)$ & $(8,8)$ & $(9,9)$ & $(10,10)$ & \\
\hline Diameter, Å & 8.2 & 9.6 & 10.9 & 12.2 & 13.6 & \\
\hline
\end{tabular}

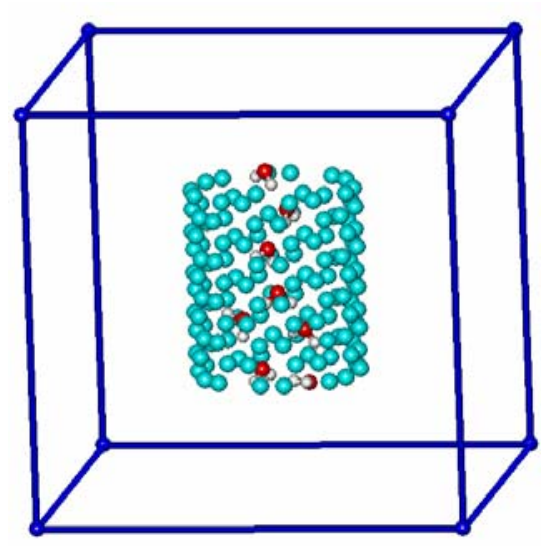

$\boldsymbol{a}$

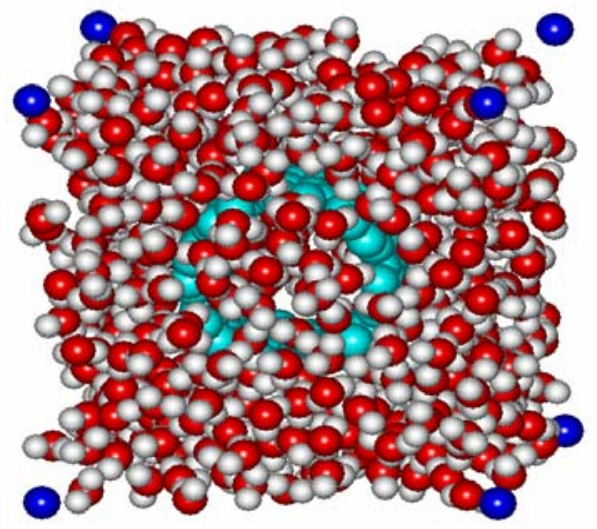

b

Figure 2. Elementary cell containing hydrated $(8,8)$ carbon nanotube $(a)$, the same without outer water shells $(b)$.

We used a NpT thermodynamic ensemble with $\mathrm{p}=1$ bar, $\mathrm{T}=298 \mathrm{~K}$. The BMW intermolecular potential was used for calculating the intermolecular potential energy between rigid water molecules. The BMW potential offers two significant qualitative differences with any other additive potentials. In the BMW model inverted and bifurcated geometries are treated as local minima on the potential energy surface. The shape of the PES near each local minimum was approximated by Gaussian functions. As a result, we created a multi-attractor effective potential which reproduces experimental thermodynamic and structural properties of real water within a wide range state parameters.

The carbon-water Lennard-Jones (12-6) parameters were $\sigma_{\mathrm{CO}}=3.2751 \AA$ and $\varepsilon_{\mathrm{CO}}=0.478 \mathrm{~kJ} \mathrm{~mol}^{-1}$. The Markovian chains included $\sim 10^{9}$ configurations. Water-water interaction were spherically truncated at $10 \AA$. Atoms of $\mathrm{CN}$ interacted with all water molecules in the elementary cell. We used our own Monte-Carlo simulation program for all calculations.

\section{Results and discussion}

We separated out water molecules according of their location in or out CNs and we studied the structural properties. The results of investigations of the topological properties of H-bond water network for molecules in CNs are represented on Figs. 3-6. In all cases one can notice significant fluctuations in the number of water molecules inside the tubes. Results are independent on the tube chirality. There are two possible states corresponding to filled and empty in the small diameter range. 
Large diameter tubes are filled all the time. A small variation in the diameter leads to significant modifications in occupancy numbers.

Decrease in the tube diameter leads to a rather dramatic destruction of the linear H-bond water network. The mean H-bond number per molecule decreases significantly from 3.5 to 0.5 . We expect that only the $3 \mathrm{~A}$ model and the BMW potential can make such kind of predictions.

Concentrations of bifurcated dimers in the first hydration shells of carbon nanotubes increase approximately 4 times relatively to the bulk water population. The concentration of inverted dimers increases by a factor two. These results do not depend on the chirality and curvature of the tube's surface (see also, Figs. 7-9).
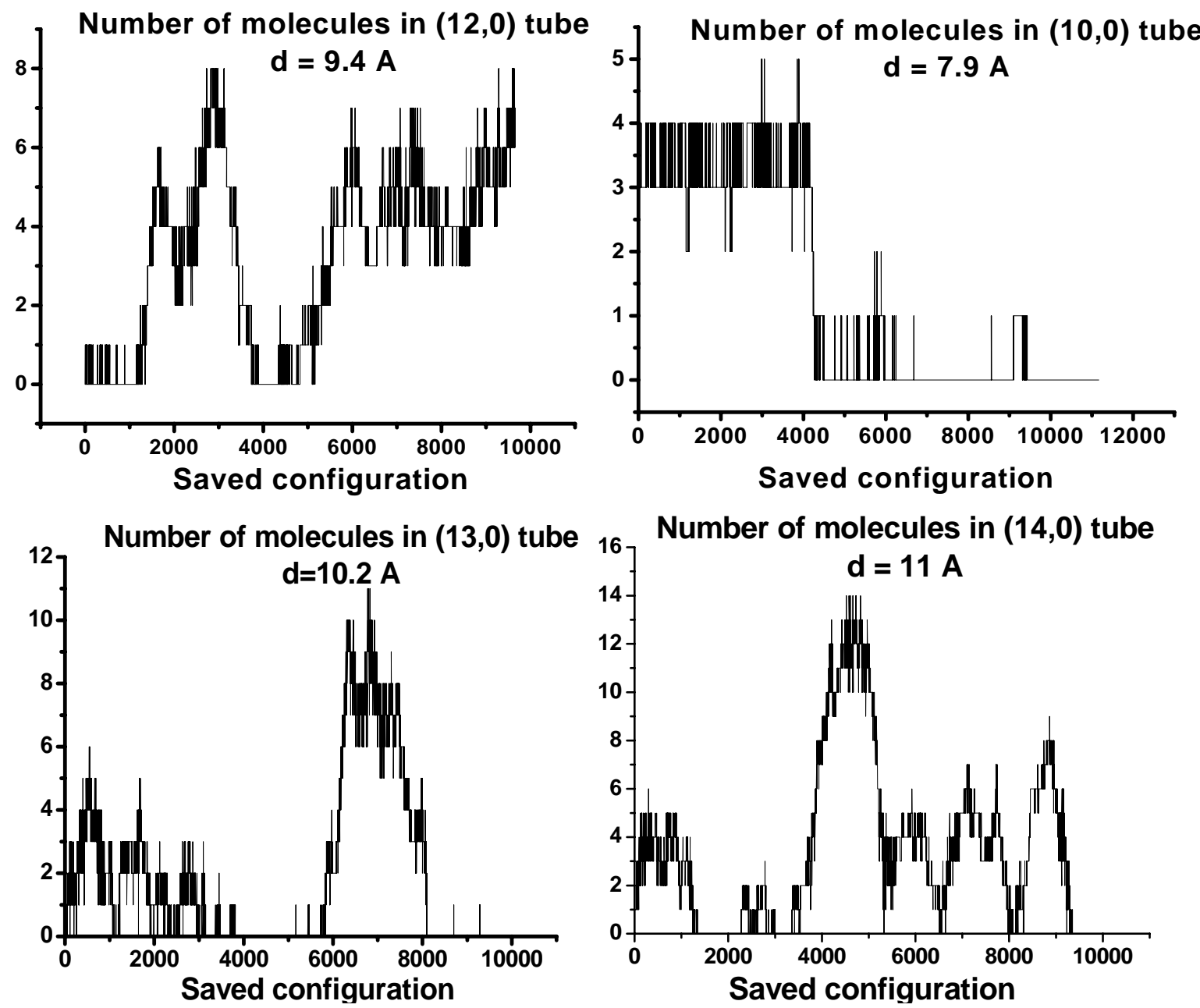

Figure 3. Dependencies of amount of inner water content on the tubes' diameters and on the length of Markovian chains. Graphics concern zigzag tubes. 

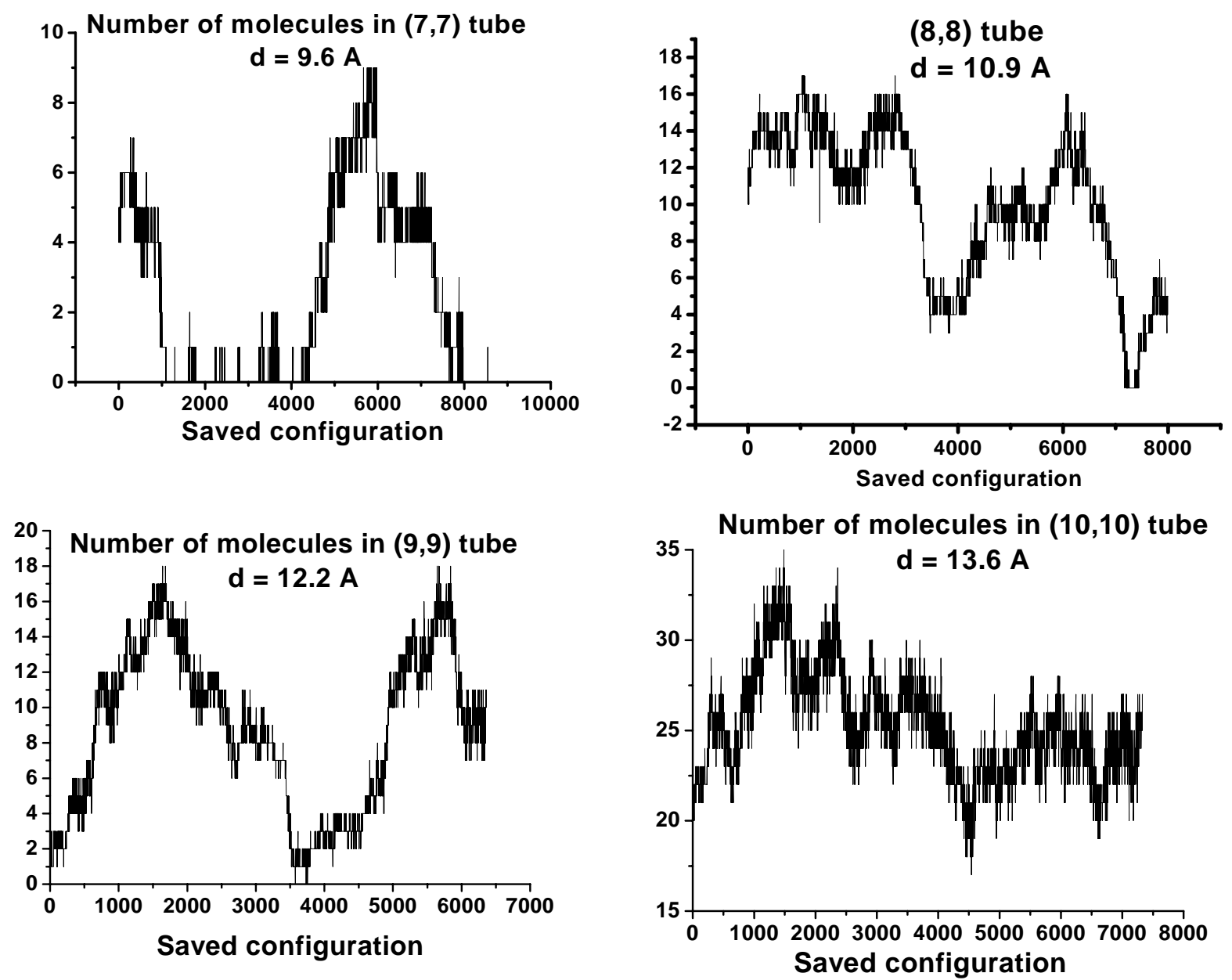

Figure 4. Similar to Fig. 3 observations concerning armchair $\mathrm{CN}$ tubes.

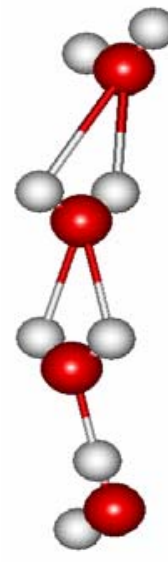

a

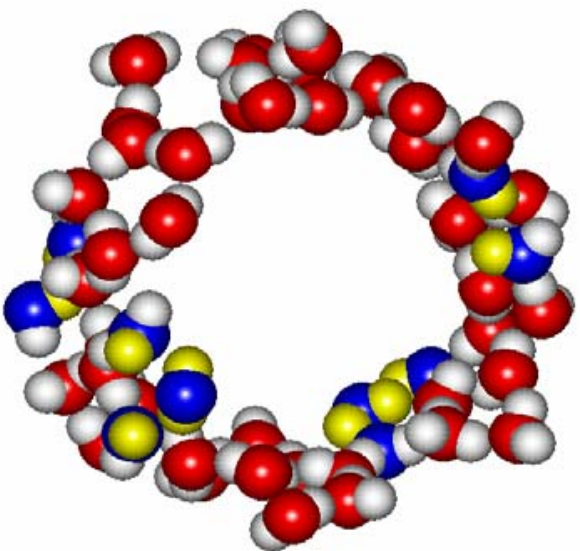

b

Figure 5. The inner $(a)$ and outer $(b)$ clusters formed by water molecules for a $(6,6)$ nanotube immersed in water. BD and ID associates in outer clusters are marked by color. $\mathrm{CN}$ is not shown. 


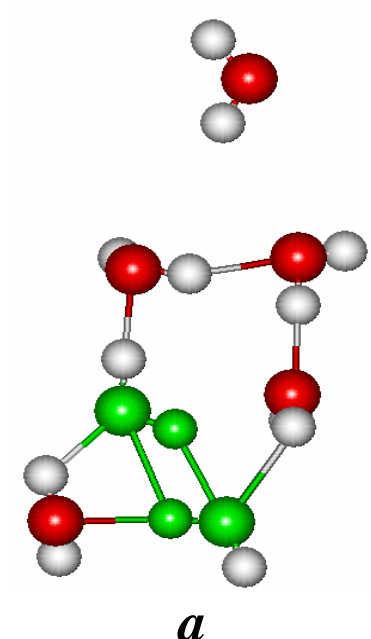

a

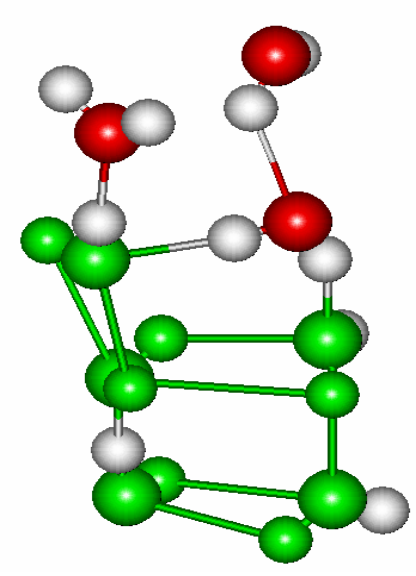

b

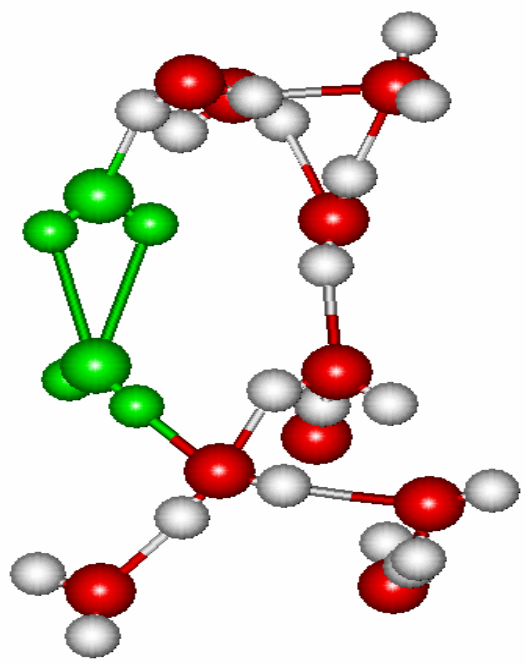

C

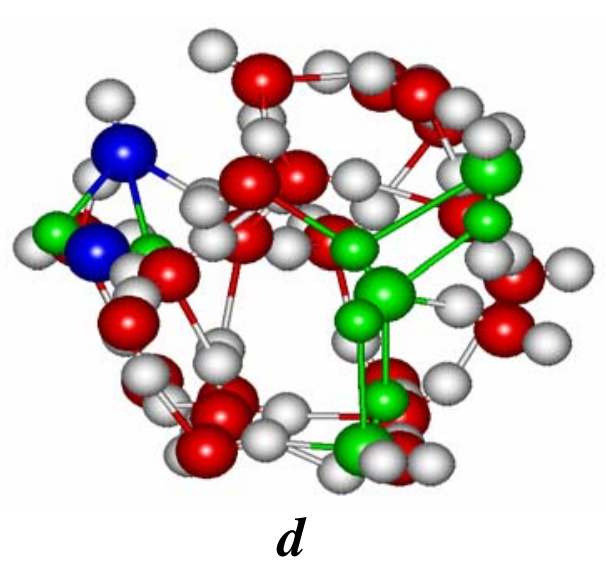

Figure 6. The inner clusters formed by water molecules for: $a, b-(8,8) ; c-(13,0) ; d-(10,10)$ nanotube immersed in water. BD and ID associates are marked by color.

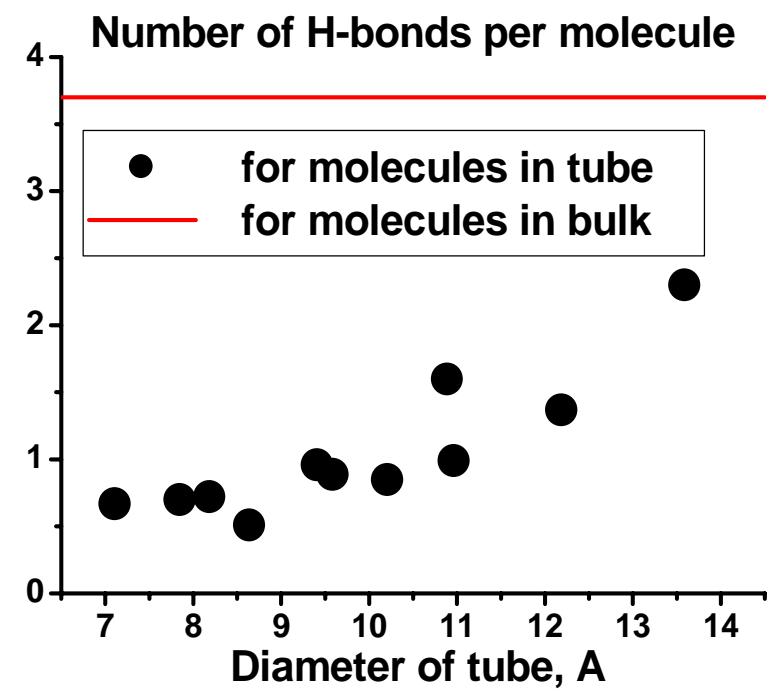

Figure 7. Dependencies of amount of $\mathrm{H}$-bonds on the $\mathrm{CN}$ diameters. 


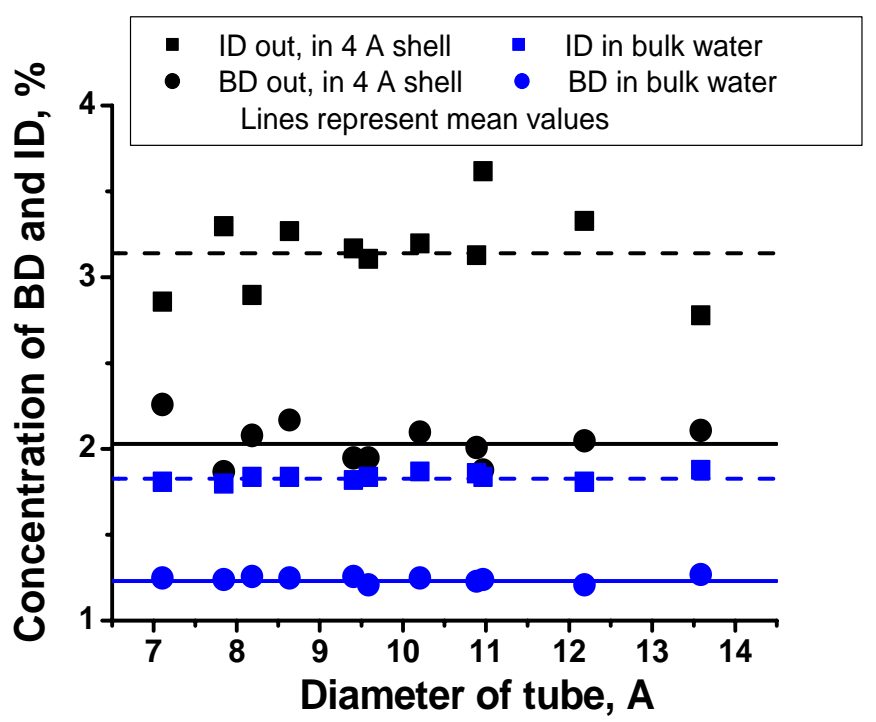

Figure 8. Dependencies of concentration of BD and ID in outer hydration shell and bulk water on the CN diameters.
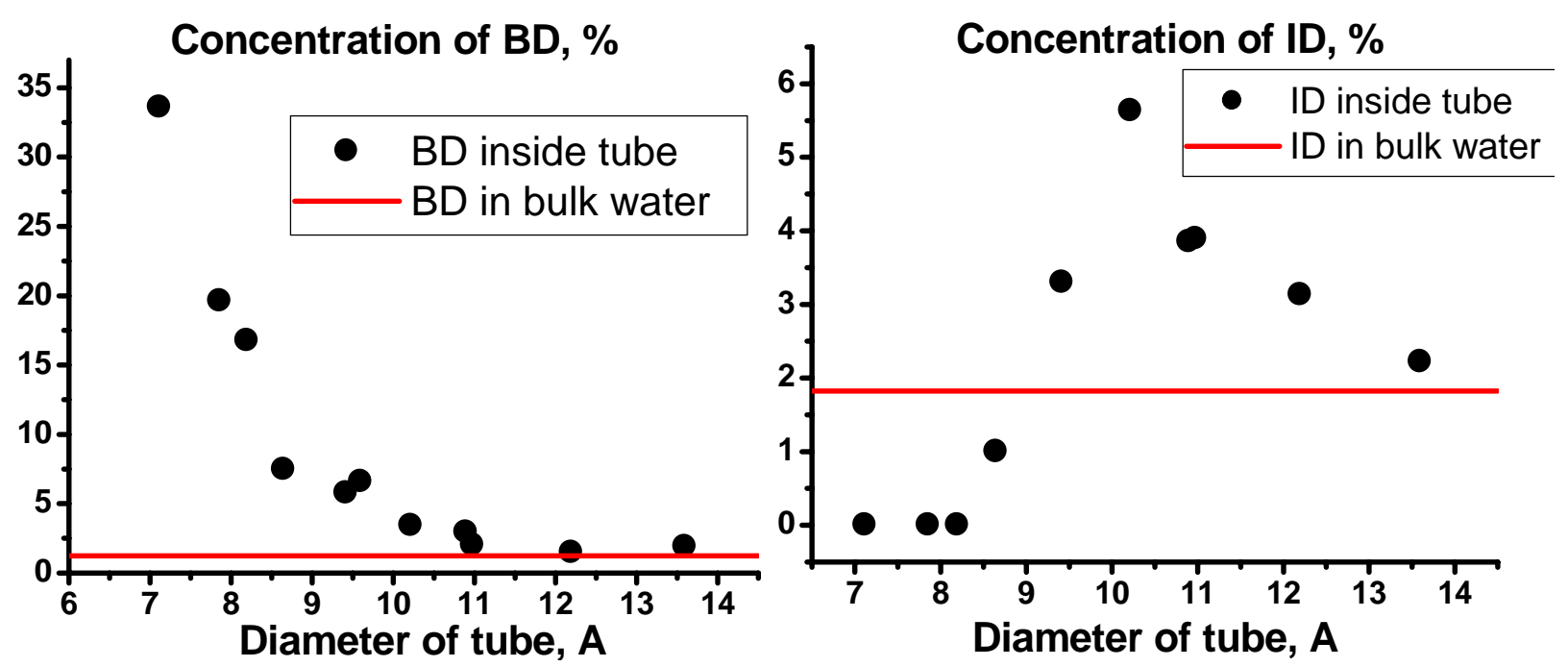

Figure 9. Dependencies of concentrations of BD and ID for inner and bulk water on the CN diameters.

The most significant changes in the H-bond network topology are observed for inner water molecules. The concentration of bifurcated dimers sharply increases while diameters decrease from 9 to $7 \AA$. As much as $30 \%$ of molecules are involved in bifurcated dimers. In the case of inverted dimers, the curve is more complicated. It has maximum for tubes diameter 10-11 $\AA$. Concentrations of inverted and bifurcated dimers tend to values corresponding to bulk water only when tubes diameter becomes larger.

\section{Conclusions}

It was found, that a carbon nanotube immersed in water modifies in an essential way the structural properties of inner and outer liquid water. Tubes occupancy and properties of inner water changes sharply and in a non-monotonic way with change of its diameter. Carbon nanotubes may provide 
wonderful research tools to investigate liquid water structure and might provide a much welcome way to validate or reject a proposed liquid water model.

Our finding indicates that much caution be exercised when designing carbon nanotube-based sensors in liquid water and aqueous solutions. Specific properties of the liquid flow through carbon nanotubes, ionic transport, and the amount of electricity being produced should be essentially dependent on tube diameters and properties of pure water liquid or aqueous solution. Hydration of solutes is likely to be also affected.

Our study also demonstrates that a complete understanding of the liquid water structure is not just a question of basic science: it is also a question of applied science and technology. It is urgently needed in order to design $\mathrm{CN}$ sensors in aqueous solutions.

\section{Acknowledgements}

This work was supported in part by a grant from the MDPI foundation with the help of the Open Society Institute" (OSI), and in part by the Russian Foundation for Basic Research, project no. 04-0332363a. We wish also to acknowledge the kind support of ISUCT, ISC and ENSTA.

\section{References and notes}

1. Ghosh, S.; Sood, A.K.; Kumar, N. Carbon nanotube flow sensors, Science 2003, 299, 1042.

2. Sotiropoulou, S.; Chaniotakis, N.A. Carbon nanotube array-based biosensor, Anal. Bioanal. Chem. 2003, 375, 103.

3. Kong, J.; Franklin, N.R.; Zhou, C.; Chapline, M.G.; Peng, S.; Dai, K.C.H. Nanotube molecular wires as chemical sensors, Science 2000, 287, 622.

4. Maiti, A.; Andzelm, J.; Tanpipat, N.; von Allmen, P. Effect of adsorbates on field-emission from carbon nanotubes, Phys. Rev. Lett. 2001, 87, 155502.

5. Hummer, G.; Rasaiah, J.C.; Noworyta, J.P. Water conduction through the hydrophobic channel of a carbon nanotube, Nature 2001, 414, 188.

6. Muguet, F.F. Investigation of diffuse intermolecular electronic systems, Ph.D. dissertation, 1992, published by UMI (Ann Arbor, MI, USA).

7. Muguet, F.F. The water dimer: symmetries and ZPE corrections according to intermolecular distance, Internet J. Chem. 2000, 3, 6.

8. Bushuev, Yu.G.; Davletbaeva, S.V.; Muguet, F.F. The 3-Attractor water model: Monte-Carlo simulations with a new, effective 2-body potential (BMW), Molecules 2003, 8, 226.

9. Berendsen, H.J.C; Grigera, J.R.; Straatsma, T.P. The missing term in effective pair potentials, J. Phys. Chem. 1987, 91, 6269.

(C) 2005 by MDPI (http://www.mdpi.net). Reproduction is permitted for non-commercial purposes. 